\title{
Tubo de Lester-Jones: Indicações e resultados
}

\author{
Lester-Jones tube:Indications and results
}

\author{
Walberto Passos Junior ${ }^{1}$ \\ Silvana Artioli Schellini ${ }^{2}$ \\ Carlos Roberto Padovani ${ }^{3}$
}

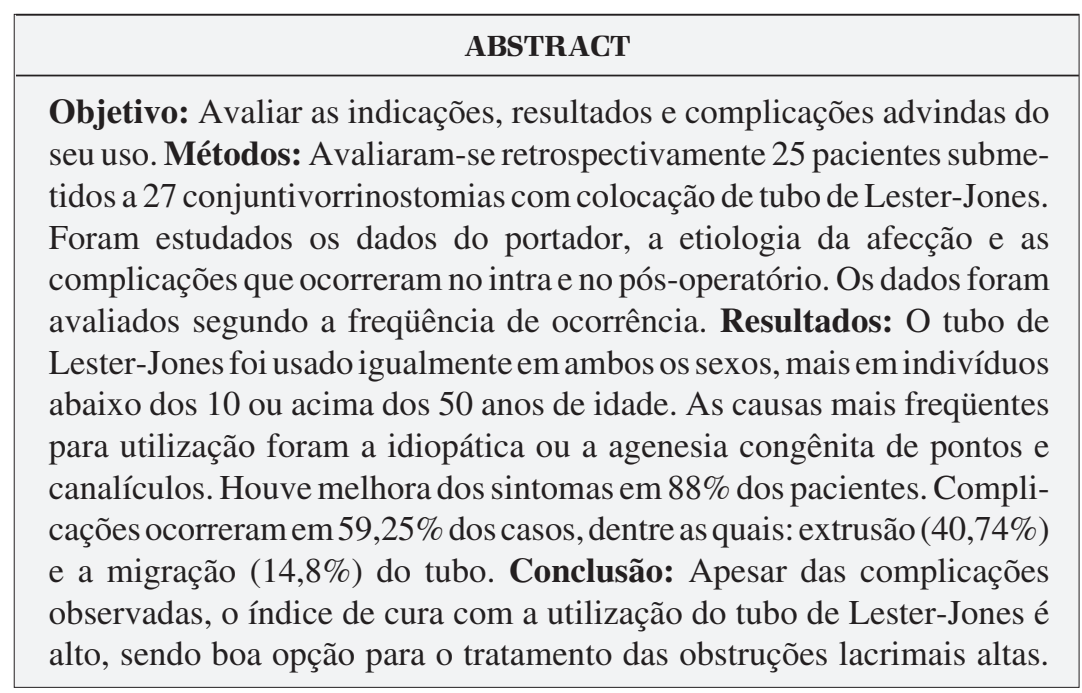

Descritores: Obstrução dos ductos lacrimais/cirurgia; Ducto nasolacrimal/cirurgia; Dacriocistorinostomia/métodos; Aparelho lacrimal/patologia; Complicações pós-operatórias

\section{INTRODUÇÃO}

O tratamento atualmente mais aceito para as obstruções lacrimais altas consiste na comunicação direta entre o lago lacrimal conjuntival e o meato nasal médio, com (conjuntivodacriocistorrinontomia) ou sem (conjuntivorrinostomia) a utilização de retalho do saco lacrimal ${ }^{(1)}$.

Apesar de alguns estudos de série de pacientes apontarem elevados índices de complicações ${ }^{(1-3)}$, o tubo de Lester-Jones ainda é o melhor recurso a ser utilizado para este fim.

O tubo de Lester-Jones é uma prótese de vidro pirex, cujo comprimento varia entre 10 e $25 \mathrm{~mm}$, com diâmetro interno capilar e diâmetro externo de 2,2 mm. Existem vários formatos e desenhos para este tubo. Porém, o mais utilizado possui na extremidade conjuntival uma dilatação, uma espécie de colarinho, enquanto a extremidade nasal, termina em bisel ${ }^{(4)}$. O colarinho pode ter diâmetros de 3 ou $4 \mathrm{~mm}$, sendo o de $3 \mathrm{~mm}$, o mais utilizado.

Este estudo foi feito com o objetivo de avaliar as indicações, bem como os resultados e as complicações observadas nos pacientes submetidos à colocação do tubo de Lester-Jones na Faculdade de Medicina de Botucatu.

\section{MÉTODOS}

Foram examinados retrospectivamente 25 pacientes submetidos a conjuntivorrinostomia com implante do tubo de Lester-Jones, operados entre 1988 e 2002, no Hospital das Clínicas da Faculdade de Medicina de Botucatu-Unesp. 
Os parâmetros de estudo foram: faixa etária dos pacientes, queixa apresentada, lateralidade, topografia e natureza da obstrução, técnica cirúrgica empregada e complicações intra e pós-operatórias.

Técnica cirúrgica (conjuntivorrinostomia): sob anestesia geral, foi realizada incisão retilínea no dorso do nariz e luxação do saco lacrimal. A seguir, procedeu-se a pequena osteotomia do osso lacrimal, abertura e remoção da mucosa nasal. Após ressecção do terço inferior da carúncula, foi feito o trajeto por onde deveria passar o tubo com tesoura de ponta reta, usada como guia para a introdução de uma sonda de Bowman que serviria para avaliar a medida do comprimento do tubo a ser utilizado (o tubo deveria ser $2 \mathrm{~mm}$ menor que a distância entre a carúncula e o septo nasal). Em seguida, foi feita a introdução e fixação do tubo com fio de Seda 6-0 à conjuntiva da porção inferior a carúncula, na região do lago lacrimal. A funcionalidade imediata do tubo foi, então, testada instilando-se soro fisiológico no lago lacrimal, aspirado pela cavidade nasal.

Nas avaliações pós-operatórias, os parâmetros para pesquisar a permeabilidade da via lacrimal excretora foram o teste de Milder e o movimento de ar e líquidos pelo tubo quando da inspiração profunda, observado em lâmpada-de-fenda.

Os dados obtidos foram submetidos à análise estatística para avaliação da freqüência de ocorrência.

\section{RESULTADOS}

No período estudado foram implantados 27 tubos em 25 pacientes, sendo 13 pacientes (52\%) do sexo masculino e 12 (48\%), do sexo feminino.

Quanto à faixa etária (Gráfico 1), as cirurgias foram mais freqüentes em pacientes abaixo dos $10(28 \%)$ e acima dos 50 (28\%) anos de idade.

Todos os pacientes eram portadores de epífora. Inflamação ocular e secreção purulenta estavam presentes entre as

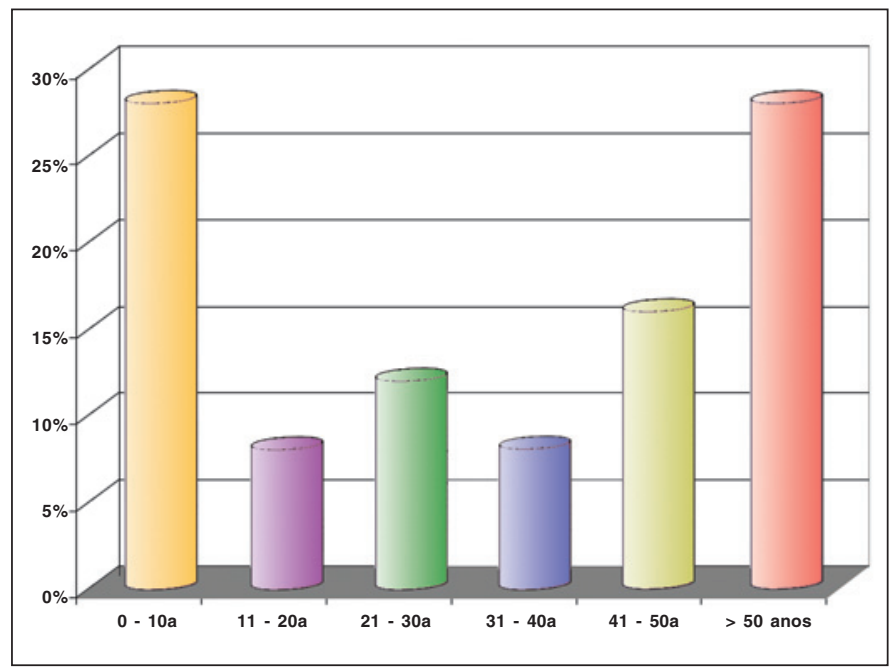

Gráfico 1 - Distribuição dos pacientes quanto à faixa etária queixas de $16 \%$ e $12 \%$ dos pacientes, respectivamente. As queixas ocorreram no olho direito em $36 \%$ e no olho esquerdo em $56 \%$ dos pacientes. Em $8 \%$, a obstrução era bilateral.

As causas de obstrução mais freqüentes foram: idiopática (28\%) e agenesia canalicular (24\%) (Gráfico 2).

O período de seguimento variou entre 12 e 60 meses, observando-se melhora dos sintomas ao final do seguimento em $88 \%$ dos pacientes.

Dentre os 27 tubos colocados, ocorreram complicações em $59,25 \%$ dos casos. Destas, as mais freqüentes foram a extrusão $(40,74 \%)$ e a migração $(14,8 \%)$ do tubo (Tabela 1$)$.

O tempo decorrido entre a cirurgia e a extrusão variou entre 15 dias a 12 meses, com média de 5,2 meses.

Dos 11 casos em que ocorreu extrusão do tubo, em 45,45\% realizou-se nova cirurgia; nos outros $54,54 \%$, não havia mais a queixa de epífora. Estes pacientes foram considerados curados, embora não se tenha realizado nenhum teste para comprovar a patência do trajeto criado para a drenagem das lágrimas.

A retirada do tubo foi efetuada em $29,62 \%$ dos pacientes. O tempo decorrido entre a cirurgia e a retirada do tubo variou de 2 a 6 meses, com média de 4,06. As causas para a retirada foram: migração do tubo em 4 casos, mal-posicionamento causando incômodo ao paciente (sensação de corpo estranho) em 2 casos, extrusão parcial em 1 e abscesso etmoidal em outro caso.

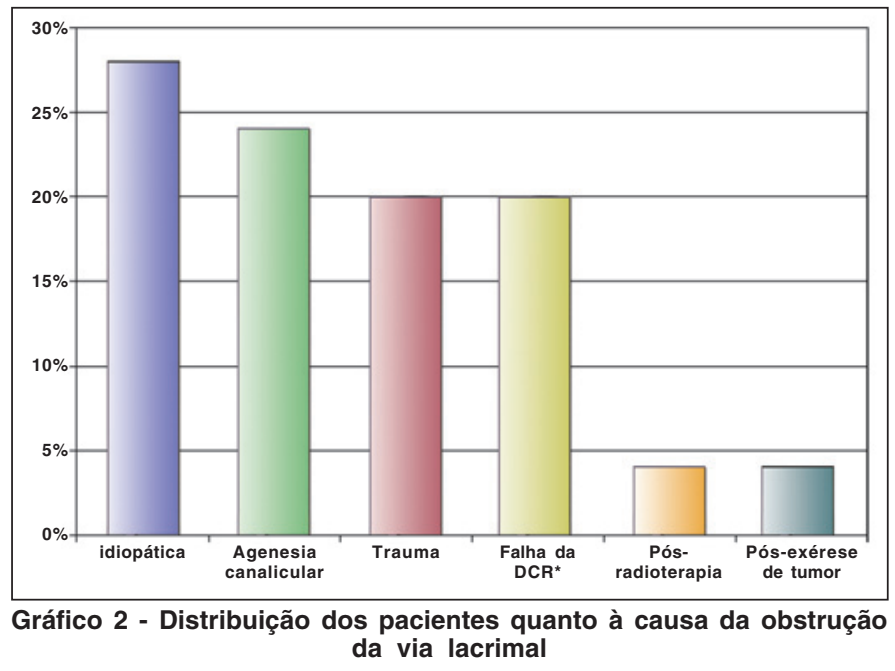

\begin{tabular}{|c|c|}
\hline Complicações & Freqüência* \\
\hline Extrusão & $11(40,74 \%)$ \\
\hline Migração & $4(14,8 \%)$ \\
\hline Inflamação conjuntival & $2 \quad(7,4 \%)$ \\
\hline Redundância conjuntival & $2 \quad(7,4 \%)$ \\
\hline Mal-posicionamento & $2 \quad(7,4 \%)$ \\
\hline Granuloma conjuntival & $1 \quad(3,7 \%)$ \\
\hline Abscesso etmoidal & $1 \quad(3,7 \%)$ \\
\hline
\end{tabular}




\section{DISCUSSÃO}

A colocação do tubo de Lester-Jones é um procedimento que possui indicações precisas, sendo raros os relatos que envolvem série numerosa de pacientes. Uma das maiores séries possui 310 pacientes, avaliados em intervalo de 25 anos, ou seja, com média de 12,4 cirurgias por ano ${ }^{(3)}$.

No presente estudo, a maioria dos indivíduos que receberam o tubo de Lester-Jones possuía menos do que 10 ou mais do que 50 anos de idade, isso porque as causas que levaram a necessidade do procedimento incidiram mais nestas faixas etárias.

A agenesia da via lacrimal (pontos e/ou canalículos) é uma causa freqüente que leva ao procedimento. Além desta, outras causas de obstrução alta seriam: trauma dos canalículos, infecções, doenças muco-sinequiantes, iatrogênicas (radioterapia, sondagens, medicamentosas) e idiopáticas ${ }^{(5)}$. A necessidade de se colocar o tubo de Lester-Jones em indivíduos submetidos a dacriocistorrinostomia ocorre por insucesso do procedimento, causada por fibrose excessiva, com oclusão da comunicação entre o saco lacrimal e a cavidade nasal.

As causas de obstrução alta diferem nas séries de casos apresentadas, dependendo de características dos serviços em que os estudos são realizados. Assim, o trauma aparece como importante causa para alguns ${ }^{1}$, a infecção herpética para ou$\operatorname{tros}^{(6)}$. Observa-se também a causa iatrogênica, destacandose a conseqüente a quimioterapia ${ }^{(6)}$.

Dos pacientes aqui estudados, a agenesia canalicular era de apenas um dos canalículos em 66,6\% dos casos. Ainda assim, havia epífora e os pacientes foram beneficiados com a cirurgia, como também observado por outros ${ }^{(7)}$.

A recanalização para a drenagem das lágrimas é possível mediante o cuidadoso posicionamento do pequeno tubo, colocado entre o lago lacrimal, na região da carúncula, e o meato nasal médio.

A técnica cirúrgica empregada foi a mesma (conjuntivorrinostomia) em todos os casos. Existem muitas variantes da técnica, utilizando-se retalhos do saco lacrimal e/ou mucosa nasal (conjuntivodacriocistorrinostomia), retalhos de conjuntiva, veia, artéria ou mucosa bucal ${ }^{(8-9)}$. Descreve-se, ainda, a conjuntivobucostomia, com abertura do fundo-de-saco conjuntival para o vestíbulo bucal ${ }^{(1,2,4)}$.

Mais recentemente, e relacionada com índices superiores de sucesso, surgiu a conjuntivorrinostomia endoscópica. Cita-se como uma vantagem em relação à técnica tradicional o fato de não se necessitar do acesso externo, eliminando a cicatriz do dorso nasal ${ }^{(5)}$. Porém, a nosso ver, a vantagem principal de se utilizar a endoscopia está na perfeita visibilização da extremidade distal do tubo e a colocação do mesmo na posição mais adequada, já que as principais complicações decorrentes do procedimento se relacionam com a posição inadequada e extrusão do tubo ${ }^{(10)}$.

O alívio da epífora foi obtido em $88,0 \%$ dos pacientes aqui estudados, apesar de alguns terem sido submetidos a mais de uma cirurgia ou a retirada do tubo. Embora não se tenha realizado provas para avaliação anatômica do trajeto fistuloso, os pacientes foram considerados curados por não apresentarem mais epífora.

Índices de sucesso semelhantes ou superiores foram apontados por outros ${ }^{(8)}$, com chance de alívio da epífora em 97,0\% ${ }^{(11)}$ ou $98,5 \%{ }^{(1)}$ após a colocação do tubo de Lester-Jones.

Apesar do alto índice de sucesso, é comum se observar uma alta taxa de complicações, destacando-se extrusão, migração, posicionamento inadequado, obstrução, granuloma conjuntival, infecção, episclerite, erosão escleral e diplopia ${ }^{(1,10-11)}$.

A extrusão e a migração são facilitadas por trajetos fistulosos amplos, sendo aconselhável realizar osteotomia pequena e trajetos estreitos para a passagem do tubo.

A escolha do tamanho adequado do tubo também pode auxiliar na manutenção da posição do mesmo, uma vez que os grandes tendem a migrar mais facilmente.

O tubo de Lester-Jones tem algumas propriedades, como a de ser bem tolerado, de ter boa capilaridade, proporcionando boa drenagem lacrimal. É fácil de ser limpo, bem como, removido ou reposicionado.

Embora o tubo de Lester-Jones seja considerado uma prótese definitiva, por vezes, pode ser necessária a sua retirada ou o seu reposicionamento, o que se observou em $29,62 \%$ dos casos, ocorrendo, em média, 4,06 meses após a cirurgia. Este tempo pode ser suficiente para que haja um trajeto formado e que se mantém patente para o carreamento das lágrimas, ficando o paciente livre da epífora.

Se a epífora se mantém, pode ser necessária nova colocação do tubo, o que ocorreu em $44 \%$ dos casos de outra série estudada, havendo resultado satisfatório em $91 \%$ dos pacientes ${ }^{(3)}$.

Houve granuloma conjuntival em 1 dos pacientes $(3,7 \%)$ deste estudo, tratado com exérese cirúrgica, não havendo recidiva do mesmo. O granuloma, em geral, decorre do fio-desutura, colocado para fixação do tubo. É facilmente removido e não causa maiores problemas.

Mesmo que ocorra a resolução da epífora com a colocação do tubo, o paciente pode continuar insatisfeito com o resultado. Esta foi a constatação de um estudo, onde $11,2 \%$ dos indivíduos com tubo funcionante estavam insatisfeitos ${ }^{(12)}$, sendo aconselhável a realização do procedimento apenas nos pacientes realmente sintomáticos e após esclarecimento quanto aos riscos de complicações.

Apesar das freqüentes complicações, a conjuntivorrinostomia com colocação do tubo de Lester-Jones apresenta alta taxa de sucesso funcional, constituindo-se, no momento, na melhor opção de tratamento das obstruções altas da via lacrimal excretora.

\section{ABSTRACT}

Purpose: To evaluate the results and the complications occurring with this procedure. Methods: The authors retrospectively reviewed charts of 25 patients (27 lacrimal viers) with upper lacrimal system obstruction who underwent conjunctivorhinostomy and Lester-Jones tube bypass. The patients were evaluated according to gender, causative factors and the com- 
plications during and after surgery. The data were submitted to statistical analysis. Results: The Lester-Jones bypass tube was used in both sexes and most frequently in patients under 10 or above 50 years. The most common obstructive factor was unknown and congenital agenesis. Eighty-eight per cent of the patients had no epiphora at the end of the treatment. Complications occurred in $59.25 \%$ of the patients, most of them related to tube extrusion $(40.74 \%)$ or migration $(14.8 \%)$. The authors concluded that the Lester-Jones bypass tube is a good option to treat the upper lacrimal obstruction, in spite of the observed complications.

Keywords: Lacrimal duct obstruction; Nasolacrimal duct/ surgery; Dacryocystorhinostomy/methods; Lacrimal apparatus/ pathology; Postoperative complications

\section{REFERÊNCIAS}

1. Sekhar GC, Dortzbach RK, Gonnering RS, Lemke BN. Problems associated with conjunctivodacryocystorhinostomy. Am J Ophthalmol. 1991;112(5):502-6.
2. Nano-Costa M, Schellini SA, Moura EM. Conjuntivorrinostomia. In: Manual de cirurgia plástica ocular. São Paulo: Roca. 2003. p.263-70.

3. Rose GE, Welham RA. Jones' lacrimal canalicular bypass tubes: twenty-five years' experience. Eye. 1991;5(Pt 1):13-9.

4. Jones LT. The cure of epiphora due to canalicular disorders, trauma and surgical failures on the lacrimal passages. Trans Am Acad Ophthalmol Otolaryngol. 1962;66:506-24.

5. Trotter WL, Meyer DR. Endoscopic conjunctivodacryocystorhinostomy with Jones tube placement. Ophthalmology. 2000;107(6):1206-9.

6. Rumelt S. Blind canalicular marsupialization in complete punctal absence as part of a systematic approach for classification and treatment of lacrimal system obstructions. Plast Reconstr Surg. 2003;112(2):396-403.

7. Lyons CJ, Rosser PM, Welham RA. The management of punctal agenesis. Ophthalmology. 1993;100(12):1851-5.

8. Campbell CB 3rd, Shannon GM, Flanagan JC. Conjunctivodacryocystorhinostomy with mucous membrane graft. Ophthalmic Surg. 1983;14(8):647-52.

9. Leone CR Jr. Conjunctivodacryocystorhinostomy with buccal mucosal graft. Arch Ophthalmol. 1995;113(1):113-5.

10. Hannas TR, Soares EJC, França VP, Figueiredo ARP, Bedran EGM. Problemas associados com a conjuntivo-dacriocistorrionostomia. Rev Bras Oftalmol. 1995;54(4): 57-61.

11. Steinsapir KD, Glatt HJ, Putterman AM. A 16-year study of conjunctival dacryocystorhinostomy. Am J Ophthalmol. 1990;109(4):387-93.

12. Rosen N, Ashkenazi I, Rosner M. Patient dissatisfaction after functionally successful conjunctivodacryocystorhinostomy with Jones tube. Am J Ophthalmol. 1994;117(5):636-42. 